\title{
The COVID-19 Pandemic: Links Between COVID-19 Complications and its Hematological Findings and Insights to Stem Cell Therapy
}

\author{
Sherif A.A. Mohamed ${ }^{1 *}$, Ghada El-Gohary ${ }^{2,3}$, Farjah AlGahtani ${ }^{2}$, Fakher Alayoubi ${ }^{4}$, \\ Nashwa Abd El-Aziz ${ }^{2,5}$ \\ ${ }^{\prime}$ Department of Chest Diseases and Tuberculosis, Faculty of Medicine, Assiut University, Assiut, Egypt. \\ ${ }^{2}$ Division of Hematology/Oncology, College of Medicine, King Saud Medical City King Saud University, Riyadh, Saudi Arabia. \\ ${ }^{3}$ Departement of Adult Hematology/Internal Medicine, Faculty of Medicine, Ain Shams University, Cairo, Egypt. \\ ${ }^{4}$ Intensive Cardiology Clinical pharmacist, King Fahd Cardic Center, College of Pharmacy, King Saud University, Riyadh, Saudia Arabia. \\ ${ }^{5}$ Department of Medical Oncology, South Egypt Cancer Institute, Assiut University, Assiut, Egypt.
}

Correspondins Author Information

Sherif A.A. Mohamed

Department of Chest Diseases and Tuberculosis, Faculty of Medicine, Assiut University, Assiut, Egypt.

Received: June 04, 2020; Accepted: June 30, 2020; Published: July 10, 2020

Copyright: (C) 2020 ASRJS. This is an openaccess article distributed under the terms of the Creative Commons Attribution 4.0 International license.

Citation: Sherif A.A. Mohamed. The COVID-19 Pandemic: Links Between COVID-19 Complications and its Hematological Findings and Insights to Stem Cell Therapy. Med Clin Res Open Access, 2020;1:1-6.

\section{Introduction}

The coronavirus infection COVID-19 first presented as an outbreak of atypical pneumonia in Wuhan, China on December 12, 2019 [1]. Since then it has spread globally to infect over 6 million cases, by the end of May, 2020. This pandemic has impacted health and the economy worldwide on an unprecedented scale. As the pandemic sweeps all over the world, clinicians start to realize that, whereas COVID-19 is primarily a respiratory infection, it has important systemic effects including the cardiovascular and immune systems. Indeed, COVI-19 is associated with distinct hematological changes, rise in serum inflammatory markers, and coagulopathy. More importantly, most of these changes were related to the patients' prognosis and mortality, particularly in those with severe disease. While we are learning constantly about the changing epidemiology, the rapidly evolving underlying science, together with insights from previous coronavirus infections, such as SARS and MERS-COV, can help us to better understand COVID-19, and in turn diagnose and treat our patients more efficiently.

In this article, we discuss the links between COVID-19 clinical features and complications and its hematological findings and coagulopathy. This link may shed light on our prognostic view to patients with COVID-19 and will have significant therapeutic implications. One of these important implications is the use of mesenchymal stem cells (MSCs) to treat COVID-19 patients. Herein, the role of this kind of novel therapy will be discussed, as well.

\section{COVID-19 virology}

SARS-CoV-2, the virus causing COVID-19, is a novel betacoronavirus (large RNA virus) that shares $80 \%$ sequence homology with the earlier SARS-CoV virus that caused the SARS 
outbreak in 2003 [2]. However, SARS-CoV-2 has evolved several features that make it a more efficient than SARS-CoV. The most critical receptor binding domain of SARS-CoV-2 preserved the overall configuration of the SARS-CoV binding domain, including 8 of the 14 residues being completely identical [3]. However, the $3 \mathrm{D}$ structure of the SARS-CoV-2 binding site shows that it is more compact, has improved binding stability, and potentially enhanced ACE-2 receptor binding affinity [3]. Another important difference is that SARS-CoV-2 contains a polybasic (furin) cleavage site inserted at the boundary of the S1/S2 subunits of the spike S-protein $[4,5]$. This furin binding site is a feature shared by several recent highly pathogenic, viruses including avian influenza, and can enhance the virus' ability to internalize into cells.

\section{The ACE-2 receptor}

ACE-2 has been confirmed recently as the SARS-CoV-2 internalization receptor causing COVID-19 [5], in concert with the host's TMPRSS2 membrane protease that primes the spike $S$ protein of the virus to facilitate its cell entry [6]. ACE-2 is the same functional receptor of the earlier SARS-CoV-1, however, the presence of TMPRSS2 significantly enhances viral infectivity [7]. It seems that protease inhibitors against TMPRSS2 can block effectively viral entry and infection of lung cells in vitro.

Interestingly, it was observed that ACE-2 has important immunemodulatory actions. ACE- 2 can directly interact with macrophages in the setting of vascular and lung inflammation, as demonstrated by genetic manipulation in a model of SARS, as well as by the salutary anti-inflammatory effects of infusion of recombinant ACE-2 [8]. Indeed, ACE-2 reduces the levels of angiotensin II, which has pro-inflammatory and pro-oxidant effects. Therefore, ACE-2 is important in controlling excess systemic inflammation in the presence of danger signals [8].

As TMPRSS2 and ACE-2 facilitate SAR-CoV-2 entry, the copresence of these two molecular entities in tissues explains, to a large extent, the tropism of viral proliferation. TMPRSS2 and ACE2 are co-expressed in lung, heart, gut smooth muscle, kidney, liver, neurons and immune cells [9]. Thus, their distribution may explain the patients' systemic symptoms and laboratory findings observed in COVID-19. Interestingly, circulating ACE-2 levels in patients is sex dependent, being $50 \%$ higher in males than in females with heart failure [10]. Another intriguing association is the fact that in COVID-19 infections the death rate of males is much higher, compared to females, despite adjustment for differences in risk factor profiles [11].

\section{Clinical, haematological and immunological findings}

It was observed that SARS-CoV-2 infection can generate a diverse range of responses in patients, ranging from completely asymptomatic virus shedding to a severe inflammatory response including cytokine storm-like outcomes that are accompanied by high mortality. Our current knowledge is that $81 \%$ of infected individuals have mild symptoms, $14 \%$ have severe symptoms requiring hospitalization, while $5 \%$ become critically ill requiring mechanical ventilation. These differences in response are likely the result of many factors including; the degree of viral load, host immune response, age of the patient and the presence of co-morbidities. As per published recent data, there are 5 clinical phenotypes of COVID-19. Those phenotypes have distinct clinical presentations, prognostic features, and consequently, different management strategies [12]. Consequently, these different phenotypes could lead us to question the concept of, are we in need for "phenotype-based tailored management"?

On the extreme border, in patients with severe COVID-19, the "Cytokine storm" has been recently described, and was characterized by increased plasma concentrations of interleukins 2 (IL- 2), IL-7, IL-10, G-CSF, interferon- $\gamma$-inducible protein 10, monocyte chemoattractant protein 1, macrophage inflammatory protein 1 alpha, and tumor necrosis factor $\alpha(\mathrm{TNF}-\alpha)$. This storm is the main mechanism for ARDS responsible for mortality in patients with severe COVID-19 [13].

Notably, this severe form of COVID-19 share several clinical and laboratory features with four entities gathered under the term "hyper-ferritinemic syndrome" and including macrophage activation syndrome (MAS), adult onset Still's disease (AOSD), catastrophic anti -phospholipid syndrome (CAPS) and septic shock. It is believed that COVID-19 systemic inflammation is part of the spectrum of hyerferritinemic syndromes. This common pathogenic background underlying these conditions is a suggestion for the use of therapies that target crucial inflammatory mediators. To date, several clinical trials evaluating the efficacy of IL-6 inhibition by Tocilizumab or Sarilumab, and IL-1 inhibition by Anakinra, are ongoing [14].

In a parallel with the SARS-CoV- 1 infection, in which lymphopenia was also observed to be highly prognostic, reports of SARS-CoV-2 infections had shown an early reduction in T cells, in particular a reduction in CD4+ more than CD8+ T cells [15], then recovery of lymphocyte count coincided with clinical improvement.

The important role of $\mathrm{CD} 4+\mathrm{T}$ cells was further delineated in a primary infection model with SARS-CoV in senescent mice. $\mathrm{CD} 4+\mathrm{T}$ cells were found to enable production of neutralizing antibodies and a balanced immune response. Without CD4+ $\mathrm{T}$ cells, there was much more severe interstitial pneumonitis. When both $\mathrm{CD} 4+$ and $\mathrm{CD} 8+\mathrm{T}$ cells were depleted, there was a predominance of neutrophils and innate immune macrophages [16]. Accompanying the loss of CD4+ T cells, there was an unusual macrophage predominance in SARS-related lung infiltration. This can be accompanied by hemophagocytosis in the lung and spleen, compatible with severe immune cytokine dysregulation [16]. Prolonged virus shedding in many individuals is considered as another indication that SARS-Cov-2 induces a relatively mild immune response. Virus proliferation is extremely rapid in COVID-19, yet many patients are asymptomatic, which suggests that while the immune system is mounting a response, it is not adequate to attenuate viral replication potential.

Dynamic changes in peripheral lymphocyte subset seem to play a role in patients with COVID-19. It was observed that, total Pages 2 of 6 
lymphocytes, CD4+ T cells, CD8+ T cells, $\mathrm{B}$ cells, and natural killer (NK) cells decreased in those patients, severe cases had a lower level than mild cases [17]. The subsets showed a significant association with inflammatory status in COVID-19, especially $\mathrm{CD} 8+\mathrm{T}$ cells and $\mathrm{CD} 4+/ \mathrm{CD} 8+$ ratio. After treatment, $67 \%$ of patients showed a clinical response, with an increase in CD8+ T cells and $\mathrm{B}$ cells. In multivariate analysis, post-treatment decrease in CD8+ T cells and B cells and increase in CD4+/CD8+ ratio were indicated as independent predictors of poor efficacy [17]. Also, it was observed that, by comparison of leukomonocytes counts in COVID-19 patients at different stages of the disease, CD3+, CD4+, CD8+ T cells and B cells appear to play important roles in viral clearance. The restoration of leukomonocytes counts from peripheral blood can be used as prognosis for the recovery of an COVID-19 infection. Even, it was suggested that restoration of leukomonocytes counts can be added to the COVID-19 diagnostic guidance as a criterion for releasing patients [17].

Several Studies have shown that the fatal myocardial injury among hospitalized patients with COVID-19 was related to marked thrombocytopenia. A meta-analysis of nine studies has suggested that thrombocytopenia is significantly associated with the severity of the COVID-19 disease [18].

\section{The emerging role of biomarkers}

C-reactive protein (CRP) was found to be elevated in most of COVID-19 patients, together with elevated procalcitonin and elevated lactate dehydrogenase (LDH), which may also be suggestive of a secondary bacterial infection complicating the clinical course of COVID-19 disease. More severe cases showed a more marked increase compared with the non-severe ones [13].

In the literature, increased LDH has been associated with higher risk of acute respiratory distress syndrome (ARDS), intensive care unit (ICU) support and death [13,19]. Another emerging biomarker for COVID-19 course is interleukin-6 (IL-6). Increased IL-6 levels was associated with increased risk of death, and a gradual increase during hospitalization has been reported in non-survivors $[13,19]$.

Vascular endothelium, thrombosis, and coagulopathy

Two major findings support that SARS-CoV-2 virus targets the endothelium, one of the largest organs in the human body. First, ACE-2 is also expressed by endothelial cells [20]. Second, major clinical events were observed in COVID-19 patients (e.g., thromboembolic disease, high blood pressure, kidney injury, pulmonary embolism, and cerebrovascular stroke [20,21].

Back to physiology, the endothelium prevents blood clotting by two mechanisms. First is by providing an antithrombotic surface, maintained by heparan sulphate present in the matrix surrounding the cells through the expression of the tissue factor inhibitor, thrombomodulin. Second, through promoting fibrinolysis via the production of tissue-type plasminogen activator [21-23]. On the other side, we identify endothelial dysfunction as a systemic condition in which the endothelium loses its physiological properties, including the tendency to promote vasodilation, fibrinolysis, as well as anti-aggregation [23]. Characteristically, it was observed that patients with COVID19 often have clotting disorders, with manifest organ dysfunction and coagulopathy, resulting in higher mortality [24,25]. Analysis of coagulation profiles of samples collected from COVID-19 infected patients showed that non-survivors had significantly higher D-dimer and FDP levels, and longer PT vs. survivors [26]. Moreover, the clinical diagnosis of disseminated intravascular coagulation (DIC) was observed in non-survivors during late stages of hospitalization $[19,26]$. As expected, if DIC is attributable to systemic infection, it features an acute systemic over-inflammatory response, tightly linked to endothelial dysfunction.

Equally important, angiotensin II level in the plasma of COVID-19 patients was markedly elevated and linearly related to viral load and lung injury; notably, angiotensin II is known to increase microvascular permeability, to induce the transcription of tissue factor in endothelial cells, and to activate platelets [27,28].

Moreover, angiotensin II can trigger the release of several components of the complement system from endothelial cells, further confirming the key role of endothelium in the pathogenesis of venous and arterial thrombotic phenomena in COVID-19 patients [26-28]. In late stages of COVID-19, a dysregulated immune response is observed and plays a crucial role in endothelial dysfunction and thrombosis $[28,29]$. Quantitavely, endothelial cells represent one-third of the cell population of the lung, and physiologically, pulmonary endothelium represents a fundamental protective barrier between the blood and the interstitial space. Therefore, it is not surprising that pulmonary endothelial damage is considered the hallmark of ARDS [26-29].

The interaction between endothelial and immune cells could play a crucial role in COVID-19, especially in severe cases and in the late stages of the disease $[12,13]$. The cytokine storm might lead to abrupt hyperinflammatory and hypercoagulability states. Thus, increased vulnerability of patients with CVD and/or diabetes might simply reflect the impact of this underlying chronic inflammation and its response during SARS-CoV-2 infection. If this is the scinario, then endothelial alterations could then be seen as an epiphenomenon.

Acute pulmonary embolism (PE), reported in COVID-19 patients [29], has been shown to be a cause of clinical deterioration in viral pneumonias, as well. Clinical implications for these observations denote that, it is important to select COVID-19 patients at higher risk of $\mathrm{PE}$, and practice computed tomography pulmonary angiography (CTPA) for the diagnosis of pulmonary thromboembolism especially in case of significant increase of $\mathrm{D}$-dimer values. Anticoagulation could be a necessary therapy to control and reduce pro-thrombotic events, as well as to prevent PE [26-30].

\section{Autopsy studies}

A group of articles have been published recently for autopsy studies performed for COVID-19 patients. Ackermann and colleagues [31] 
had examined 7 lungs obtained during autopsy from patients who died from COVID-19 and compared them with 7 lungs obtained during autopsy from patients who died from acute respiratory distress syndrome (ARDS) secondary to influenza A(H1N1) infection and 10 age-matched, uninfected control lungs. They found the pattern of diffuse alveolar damage with perivascular T-cell infiltration, in patients who died from COVID-19associated or influenza-associated respiratory failure. The lungs from patients with COVID-19 also showed distinctive vascular features, consisting of severe endothelial injury associated with the presence of intracellular virus and disrupted cell membranes. The pulmonary vessels of patients with COVID-19 showed widespread thrombosis and microangiopathy. Alveolar capillary microthrombi were 9 times as prevalent in patients with COVID-19 as in patients with influenza [31]. In a study from USA, autopsy findings on 23 patients with COVID-19, confirmed that COVID-19 is a systemic disease with major involvement of the lungs and heart [32]. The authors concluded that their autopsy findings support the concept that the pathogenesis of severe COVID-19 disease involves direct viral-induced injury of multiple organs, including heart and lungs, coupled with the consequences of a procoagulant state with coagulopathy.

Mesenchymal stem cells as a potential therapy for COVID-19 Mesenchymal stem cells (MSCs) can be isolated from different adult tissues, including preferably bone marrow $(\mathrm{BM})$, peripheral blood (PB) and adipose tissues (AT) (such as abdominal fat, infrapatellar fat pad, and buccal fat pad) and neonatal

birth-associated tissues, including placenta (PL), umbilical cord (UC), Warton jelly (WJ), amniotic fluid (AF), and cord blood (CB), and then stored for future possible applications [33]. They have abundant supply, differentiation potential, powerful immunoregulation, and endogenous repair mechanisms.

Support to potential use of MSCs in treating COVID-19 patients came from observations that MSCs have been identified to efficiently cure ALI/ARDS from both infectious and noninfectious causes, mediated primarily by paracrine mechanisms based on the released extracellular vesicles (EVs), such as micro-vesicles and exosomes [34]. The immune-regulation of MSCs depends mainly on modulating activation and effector function of immune cells, suppressing lung-infiltrated cells, and enhancing the resolution of pulmonary edema [35].

Back to physiology, MSCs can alter the behavior of both adaptive and innate immune cells. They can release keratinocyte growth factor, prostaglandin E2, granulocyte-macrophage colonystimulating factor, and IL- 6 and IL-13 to facilitate the phagocytosis and alternative activation of alveolar macrophage, alter the cytokine secretion profile of dendritic cell subsets, and decrease the release of interferon $\gamma$ from natural killer cells. IL-10, transforming growth factor $\beta$, and tryptophan catabolizing enzyme indoleamine 2,3-dioxygenase secreted from them can also suppress the proliferation of $\mathrm{T}$ cells and change the cytokine secretion profile of $\mathrm{T}$ cell subsets [36]. Moreover, the proliferation, differentiation, and chemotactic properties of B cells were impaired by MSCs as well. Except for the immune regulatory effects, MSCs can enhance restoration of capillary barrier, inhibit bacterial growth, and restore alveolar ATP [37]. Interestingly, all these functions mentioned above might also be effective on ARDS induced by COVID-19 infection.

Our previous experience with the therapeutic effects of MSCs on ALI/ARDS and graft -versus- host disease (GVHD), with powerful inflammatory balance give us solid proofs for the application of MSCs on other originated ALI/ARDS, like those due to COVID-19. Furthermore, we observed that MSC treatment significantly ameliorates ALI/ARDS induced by H9N2 avian influenza virus and $\mathrm{H} 5 \mathrm{~N} 1$ in mice, and even influenza virus in pig, indicating the possible efficacy of MSCs on viral ALI/ARDS [38-40]. Importantly, MSCs can cure the patients with severe refractory ARDS, who failed to improve after both standard life support measures including mechanical ventilation and additional measures including extracorporeal ventilation, pointing that MSCs could be used for serious viral ALI/ARDS [41].

It is believed that the best role for starting MSCs therapy in the treatment of COVID-19 patients, comes at the stage of those with "cytokine storm". Probably, MSC therapy can prevent the Storm release of cytokines by the immune system and promote endogenous repair by reparative properties of the stem cells [42].

After intravenous injection, part of the MSC population entraps in the lung, which often in systemic infusion it is remembered as a limitation. But here these MSCs could recover the pulmonary microenvironment, protect alveolar epithelial cells, intercept pulmonary fibrosis, and cure lung dysfunction and COVID-19 pneumonia [39-42].

Therefore, Recently, China, USA, Jordon, Iran, and several other countries have begun cell-based therapy clinical studies and some reports have been published. Some Chinese research groups triggered the clinical studies on MSCs treating critical COVID-19. In the trigged clinical trials, the inclusion criteria for severe or critical COVID-19 include respiratory rate (RR) $\geq 30$ times/min, pulse oxygen saturation $\left(\mathrm{SpO}_{2}\right)$ at rest $\leq 93 \%$, partial pressure of $\mathrm{PaO}_{2} / \mathrm{FiO}_{2} \leq 300 \mathrm{mmHg}$, requirement for mechanical ventilation, shock, etc. As of February 21, 2020, four patients with severe COVID-19 were recovered and discharged by MSCs therapy in China [41]. According to results of these initial clinical trials, it might be noteworthy to test the safety and efficacy of MSC transfusion in COVID-19 patients, especially for the severe or critical cases.

There is much superiority in using MSC therapy in comparison with other treatments, including in 1) They are easily accessible and can be isolated from various tissues such as bone marrow and adipose tissues, including in umbilical cord, dental pulp, menstrual-blood, buccal fat pad, fetal liver, etc.; 2) They are multipotent stem cells; 3) MSCs can easily expand to clinical volume in a suitable period of time; 4) MSCs can be stored for repetitive therapeutic usage; 
5) Clinical trials of MSCs so far haven't shown adverse reactions to allogeneic MSC; 6) Safety and effectiveness of MSCs have been obviously documented in several clinical trials [41-43].

However, some issues remain, despite the significant development of the stem cell-based therapy field, immunogenicity, limited cell source and ethical issue as the main limitations of this therapeutic approach have not been solved yet.

Finally, while scientists are trying incessantly to develop a vaccine for COVID-19, as well as therapeutics to treat this disease; it seems MSCs-based therapy may possibly be an ideal candidate for clinical trials or at least the combination of treatment to treat COVID-19 patients.

\section{Conclusion}

COVID-19 is associated with distinct hematological changes, rise in serum inflammatory markers, and coagulopathy. Most of these changes were related to the patients' prognosis and mortality, particularly in those with severe disease. There are links between COVID-19 clinical features and complications and its hematological findings and coagulopathy. These links could help the clinicians understand patho-biologic mechanisms implicated in this novel disease and will have significant therapeutic implications. The use of mesenchymal stem cells to treat COVID-19 patients seems to be a promising approach. Further studies are needed to clarify more clinic-pathologic links in COVID-19 that might improve outcomes of COVID-19 patients.

\section{References}

1. Guan W, Ni Z, Hu Y, et al. Clinical characteristics of coronavirus disease 2019 in China. N Engl J Med, 2020;382:1708-1720.

2. Lan J, Ge J, Yu J, et al. Structure of the SARS-CoV-2 spike receptor-binding domain bound to the ACE2 receptor. Nature, 2020;581:215-220.

3. Shang J, Ye G, Shi K, et al. Structural basis of receptor recognition by SARS-CoV-2. Nature 2020;581:221-224.

4. Coutard B, Valle C, de Lamballerie X, Canard B, Seidah NG, Decroly E: The spike glycoprotein of the new coronavirus 2019-nCoV contains a furin-like cleavage site absent in CoV of the same clade. Antiviral Res 2020;176:104742.

5. Walls AC, Park YJ, Tortorici MA, et al. Structure, Function, and Antigenicity of the SARS-CoV-2 Spike Glycoprotein. Cell 2020.

6. Hoffmann M, Kleine-Weber H, Schroeder S, et al. SARSCoV-2 Cell Entry Depends on ACE2 and TMPRSS2 and Is Blocked by a Clinically Proven Protease Inhibitor. Cell 2020.

7. Matsuyama S, Nao N, Shirato K, et al. Enhanced isolation of SARS-CoV-2 by TMPRSS2-expressing cells. Proc Natl Acad Sci U S A 2020.

8. Wang K, Gheblawi M, Oudit GY. Angiotensin Converting Enzyme 2: A Double-Edged Sword. Circ. 2020.

9. Bertram $\mathrm{S}$, Heurich $\mathrm{A}$, Lavender $\mathrm{H}$, et al. Influenza and SARS-coronavirus activating proteases TMPRSS2 and HAT are expressed at multiple sites in human respiratory and gastrointestinal tracts. PLoS One 2012;7:e35876.

10. Uri K, Fagyas M, Manyine Siket I, et al. New perspectives in the renin-angiotensin-aldosterone system (RAAS) IV: circulating ACE2 as a biomarker of systolic dysfunction in human hypertension and heart failure. PLoS One 2014;9:e87845.

11. Liu PP, Blet A, Smyth D, et al. The Science Underlying COVID-19: Implications for the Cardiovascular System. Circulation 2020.

12. Rello J, Storti E, Belliato M, et al. Clinical phenotypes of SARSCoV-2: Implications for clinicians and researchers. Eur Respir J 2020; in press.

13. Mehta P, McAuley DF, Brown M, et al. COVID-19: consider cytokine storm syndromes and immunosuppression. Lancet 2020;S0140-6736(20)30628-0.

14. Colafrancesco S, Alessandri C, Conti F, et al. COVID-19 gone bad: A new character in the spectrum of the hyperferritinemic syndrome? Autoimmunity Reviews 2020.

15. He Z, Zhao C, Dong Q, et al. Effects of severe acute respiratory syndrome (SARS) coronavirus infection on peripheral blood lymphocytes and their subsets. Int J Infect Dis 2005;9(6):323330.

16. Chen J, Lau YF, Lamirande EW, et al. Cellular immune responses to severe acute respiratory syndrome coronavirus (SARSCoV) infection in senescent BALB/c mice: CD4+ T cells are important in control of SARS-CoV infection. J Virol 2010;84(3):1289-301.

17. Wang F, Nie J, Wang $\mathrm{H}$, et al. Characteristics of Peripheral Lymphocyte Subset Alteration in COVID-19 Pneumonia. JID 2020.

18. Lippi G, Plebani M, Henry BM. Thrombocytopenia is associated with severe coronavirus disease 2019 (COVID-19) infections: A meta-analysis. Clin Chim Acta 2020;506:145148.

19. Zhou F, Yu T, Du R, et al. Clinical course and risk factors for mortality of adult inpatients with COVID-19 in Wuhan, China: a retrospective cohort study. Lancet 2020;395(10229):10541062.

20. Sardu C, Gambardella J, Morelli MB, et al. Hypertension, Thrombosis, Kidney Failure, and Diabetes: Is COVID-19 an Endothelial Disease? A Comprehensive Evaluation of Clinical and Basic Evidence J Clin Med 2020; 9: E1417.

21. Martin FA, Murphy RP, Cummins PM. Thrombomodulin and the vascular endothelium: Insights into functional, regulatory, and therapeutic aspects. Am J Physiol Circ Physiol 2013;304(12):H1585-97.

22. Zhang MD, Xiao M, Zhang S, et al. Coagulopathy and Antiphospholipid Antibodies in Patients with Covid-19. N Engl J Med 2020; 382: e38.

23. Zhou B, She J, Wang Y, et al. Venous thrombosis and arteriosclerosis obliterans of lower extremities in a very severe patient with 2019 novel coronavirus disease: A case report. J Thromb Thrombolysis 2020;1-4.

24. Tang N, Li D, Wang X, et al. Abnormal coagulation parameters are associated with poor prognosis in patients with novel coronavirus pneumonia. J Thromb Haemost 2020;18: 844-847.

25. Toshiaki Iba, Jerrold H Levy, Theodore E Warkentin, et al. 
Scientific and Standardization Committee on DIC, and the Scientific and Standardization Committee on Perioperative and Critical Care of the International Society on Thrombosis and Haemostasis. J Thromb Haemost 2019;17(11):1989-1994.

26. Dielis AW, Smid M, Spronk HM, et al. The prothrombotic paradox of hypertension: Role of the renin-angiotensin and kallikrein-kinin systems. Hypertension 2005;46(6):1236-1242.

27. Vabret N, Britton GJ, Gruber C, et al. Immunology of COVID-19: Current state of the science. Immunity 2020.

28. Maniatis NA, Orfanos SE. The endothelium in acute lung injury/acute respiratory distress syndrome. Curr Opin Crit Care 2008;14(1):22-30.

29. Poissy J, Goutay J, Caplan M, et al. Pulmonary Embolism in COVID-19 Patients: Awareness of an Increased Prevalence. Circulation 2020.

30. Tang N, Bai H, Chen X, et al. Anticoagulant treatment is associated with decreased mortality in severe coronavirus disease 2019 patients with coagulopathy. J Thromb Haemost 2020;18(5):1094-1099.

31. Ackermann M, Verleden SE, Kuehnel M, et al. Pulmonary Vascular Endothelialitis, Thrombosis, and Angiogenesis in Covid-19. NEJM 2020.

32. Buja LM, Wolf D, Zhao B, et al. Emerging spectrum of cardiopulmonary pathology of the Coronavirus disease (COVID-19): Report of three autopsies from Houston, Texas, and review of autopsy findings from other United States cities. Cardiovascular Pathology 2020.

33. Liu S, Peng D, Qiu H, et al. Mesenchymal stem cells as a potential therapy for COVID-19. Stem Cell Res Ther 2020;11(1):169.

34. Yu Fujita, Tsukasa Kadota, Jun Araya, et al. Clinical application of mesenchymal stem cell-derived extracellular vesicle-based therapeutics for inflammatory lung diseases. J Clin Med 2018;7(10):355.

35. Harrell CR, Sadikot R, Pascual J, et al. Mesenchymal stem cell-based therapy of inflammatory lung diseases: current understanding and future perspectives. Stem Cells Int 2019;2019:4236973.

36. Aggarwal S, Pittenger MF. Human mesenchymal stem cells modulate allogeneic immune cell responses. Blood 2005;105(4):1815-1822.

37. Li Y, Xu J, Shi W, et al. Mesenchymal stromal cell treatment prevents H9N2 avian influenza virus-induced acute lung injury in mice. Stem Cell Res Ther. 2016;7(1):159.

38. Chan MC, Kuok DI, Leung CY, et al. Human mesenchymal stromal cells reduce influenza a $\mathrm{H} 5 \mathrm{~N} 1$-associated acute lung injury in vitro and in vivo. Proc Natl Acad Sci U S A 2016;113(13):3621-3626.

39. Khatri M, Richardson LA, Meulia T. Mesenchymal stem cell-derived extracellular vesicles attenuate influenza virusinduced acute lung injury in a pig model. Stem Cell Res Ther 2018;9(1):17.

40. Simonson OE, Mougiakakos D, Heldring N, et al. In vivo effects of mesenchymal stromal cells in two patients with severe acute respiratory distress syndrome. Stem Cells Transl Med 2015;4(10):1199-1213.

41. Golchin A, Seyedjafari E, Ardeshirylajimi A. Mesenchymal Stem Cell Therapy for COVID-19: Present or Future. Stem Cell Reviews and Reports.

42. Leng Z, Zhu R, HouW, et al. Transplantation of ACE2Mesenchymal stem cells improves the outcome of patients with COVID-19 pneumonia. Aging and Disease 2020;11(2),216.

43. News conference for joint prevention and control measure of COVID-19 by China's State Council. 2020. 